\title{
Udo Kauß/Falco Werkentin Was ist die Auskunftsregelung des neuen Bundesverfassungsschutz-Gesetzes wert?
}

\section{Auskunftsansprüche gegenüber dem Bundesamt für "Verfassungsschutz"}

Mehr als fünf Jahre wurde in der Öffentlichkeit, und als Folge auch im Bundestag, um sog. "Sicherheitsgesetze" gestritten. Mit ihnen sollte, so das Versprechen der Bonner Koalition, dem vom Bundesverfassungsgericht im Volkszählungsurteil des Jahres 1983 erteilten Auftrag nachgekommen werden, auch die Datenverarbeitung der Geheimdienste den Anforderungen eıner grundgesetzkonformen Datenerhebung und -verarbeitung zu unterwerfen. Nach Jahren des Streits, der zu immer neuen Entwürfen führte, wurde im Windschatten der Vereinigung das "Gesetz zur Fortentwicklung der Datenverarbeitung und des Datenschutzes « am 31. Mai 1990 mit den Stimmen der Regierungsparteien und der SPD vom Bundestag verabschiedet. Seit Januar i99x ist es in Kraft.' Im einzelnen enthält dieses Artikelgesetz eine Neufassung des Bundesdatenschutz-Gesetzes (BDSG), des Gesetzes über die "Zusammenarbeit des Bundes und der Länder in Angelegenheiten des Verfassungsschutzes " (BVerfSchG) und erstmalig ein BND- und MAD-Gesetz.

Rechtspolitisch geradezu revolutionär an diesem Gesetzespaket ist der erstmalig statuierte positive Anspruch des Bürgers, auch von den Geheimdiensten erfahren zu können, ob - und wenn ja - welche Daten sie über ihn gesammelt haben. Dies ist die rechtspolitische Konsequenz des im Volkszählungsurteil ausformulierten informationellen Selbstbestimmungsrechts der Bürger. Bisher hatten die Verwaltungsgerichte allenfalls einen Anspruch des Bürgers auf ermessensfehlerfreie Entscheidung der "Verfassungsschutz «-Behörden aus den entsprechenden Bestimmungen der Datenschutzgesetze entwickelt. ${ }^{2}$ Aber auch mit einem solcher Art verkürzten Auskunftsanspruch hatte das Bundesverwaltungsgericht mit seiner Entscheidung vom 20. Februar 1990 Schluß gemacht, indem es - noch auf alter Rechtsgrundlage verkündete, daß Auskünfte dieser Ämter an die Bürger der "Natur der Sache nach “ nicht gegeben werden müssen. In den Worten des Bundesverwaltungsgerichts:

"Das Recht auf informationelle Selbstbestimmung gibt dem einzelnen kein Recht im Sinne einer absoluten unemschränkbaren Herrschaft über seme Daten. Das gilt insbesondere für Daten, die den verfassungsrechtlich legitımierten Aufgabenberetch der für den Verfassungsschutz zuständigen Behörden betreffen. Ein auf diese Daten zielender Auskunftsanspruch des Einzelnen wird ım Rabmen des Grundsatzes der Verhältnismäßigkeit bereits von Verfassung wegen durch ein staatliches Geheimbaltungsbedürnis ausgeschlossen. «3

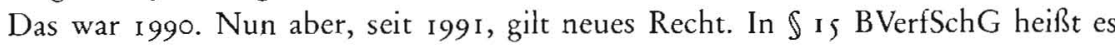
jetzt:

\footnotetext{
Bundesgesetzblatt, I, 1990, Nr.73, S. 2953 ff., nachgezerchnet ist dieser Gesetzgebungsprozeß, der exemplarısch dafür ist, was eıne kritısche Öffentlichkeıt beı entsprechendem Engagement erreıchen kann, der aber auch zeigt, wie die Bürokratie einen solchen Strett aussitzt und Situationen mangelhafter öffentlicher Aufmerksamkeıt zupackend nutzt, in folgenden Schwerpunktheften von "Bürgerrechte \& Polizet (CILIP)«: Nr. 21 (1985), Nr. 23 (1986), Nr. 29 (1988), Nr. 32 (1989), Nr. 36 (1990).

2 Vgl. mit velen Hinweisen VG Köln vom 6.5. $1988 \mathrm{~m}$ : NVwZ 1989 , S. 85 ff.

3 BVerwG, Urteil v. 20. 2. I 990 - I C 42.83 (OVG Münster), vgl. die Urteilsrezensıon von Kröger ın: Jurısten-Zeıtung 1991, 46. Jg., S. 558-563 und die geharnischte Kritik an der Entscheidung von Fuckner/ Simitis in: NJW 1990, S. 2713 ff.
} 
(1) Das Bundesamt für Verfassungsschutz erteilt dem Betroffenen über zu seiner Person gespezcherte Daten auf Antrag unentgeltlich Auskunft, soweit er hierzu auf einen konkreten Sachverhalt binweist und ein besonderes Interesse an einer Aukunft darlegt.

(2) Die Auskunftserteilung unterbleibt, sowett 1. eine Gefäbrdung der Aufgabenerfüllung durch die Auskunftserteilung zu besorgen ist,....

$\mathrm{Daß}$ dieser, beim Vorliegen der genannten gesetzlichen Voraussetzungen unbedingte Anspruch über das in Abs. 2 aufgezählte Ausnahmebündel gleichzeitig wieder begrenzt wird, entspricht üblicher Gesetzgebungstechnik.

Gewiß ist der Inhalt dieser Bestimmungen für die Geheimdienste, die »der Natur der Sache nach « im Dunklen munkeln, revolutionär. Nur waren Revolutionen noch nie das Bestreben von Geheimdiensten - schon eher der Staatsstreich.

Wie also reagierten sie auf diese rechtspolitische »Revolution«? Hält sich das Bundesamt für Verfassungsschutz an das neue Auskunftsgebot? Welche Chancen hat nun der Bürger seit dem I. Januar 199 I mit einem Auskunftsbegehren? Dieser Beitrag kann über die erste Entscheidung des Bundesamtes für Verfassungsschutz im Lichte der neuen Auskunftsregelung berichten. Sie gibt genauso wenig Hoffnung auf eine veränderte Praxis des Bundesamtes für Verfassungsschutz wie eine Stellungnahme dieser Behörde zur Auskunftsregelung im Entwurf des niedersächsischen Verfassungsschutzgesetzes vom Juni 199 I.

\section{Vorgeschichte}

Im Herbst 1988 wurde bekannt, daß das Berliner Landesamt für Verfassungsschutz sich systematisch für bestimmte Zeitungen und Journalisten interessierte. Kurzfristig setzte das Abgeordnetenhaus einen parlamentarischen Untersuchungsausschuß ein, der der Angelegenheit nachging. ${ }^{4}$ Er kam zu dem Ergebnis, daß insbesondere die »Tageszeitung « Objekt verfassungsschützerischer Begierden gewesen war. Am Rande wurde bekannt, daß auch Herausgeber der Zeıtschrift "Bürgerrechte \& Polizei (CILIP) « als linksextremistische Szenejournalisten erfaßt worden waren.

Für die Fraktion der GRÜNEN im Bundestag waren diese Erkenntnisse Anlaß, nachzufragen, ob auch das Bundesamt für Verfassungsschutz seine Neugier bestimmten Zeitschriften und Journalisten geschenkt habe. In einer parlamentarischen Antwort des Bundesministers des Inneren vom 4. April 1989 hieß es daraufhin:

"Beı der Wabrnebmung seines Auftrages, extremistische Bestrebungen zu beobachten, hat das BfV semerzett festgestellt, daß sich Mitglieder und Anbänger linksextremistzscher Organisationen an den Zettungsprojekten , die tageszettungs, , Die Neue und, CILIP, beteiligten. Solche Informationen wurden in Akten gesammelt."s

Als Mitgründer und Mitverlagsinhaber, als langjähriger Redakteur bzw. Mitarbeiter und Autoren dieser Zeitschrift hatten die Verfasser dieses Beitrags großes und, wie ihnen schien, berechtigtes Interesse, zu erfahren, wer da »ihre "Zeitschrift unterwandert. Wie hätten sie sich auch von diesen Leuten trennen können, ohne ihre Namen zu kennen? Zudem, wie hätten sie selbst den rechten Pfad verfassungs-

4 Vgl. Funk, Albrechr; Wieland, Wolfgang, Berliner Verfassungsschutz - nichts mehr so wie vorher, in: Bürgerrechte \& Polizeı (CILIP) I 989 (H.33), S. 1off.

s BT-Drs. 11/4294; vgl. auch »Verdachtsfall CILIP«, in: Bürgerrechte \& Polizes (CILIP) r989 (H. 33), S. $7 \mathrm{ff}$. 
(schutz)gefälliger Haltung wiederfinden können, wenn sie nicht erfahren würden, ob möglicherweise sie selbst von diesem abgewichen waren? Auch galt es, den durch diese amtliche Verrufserklärung ${ }^{6}$ angeschlagenen Ruf der Zeitschrift wieder herzustellen.

Eilends wurde im März 1989 - unter Verweis auf die Erklärung des Bundesinnenministers im Bundestag - durch den Anwalt der Redaktion an das Bundesamt mit der Bitte geschrieben, Auskunft darüber zu erteilen, ob von uns im Schreiben benannte Mitarbeiter bzw. Herausgeber beim Bundesamt gespeichert und darüber hinaus als Extremisten abgebucht seien. Zudem wurde das Auskunftsbegehren erweitert auch auf Sachakten, die sich auf die Zeitschrift und die Redaktion »Bürgerrechte \& Polizei (CILIP)« beziehen. Hierzu parallel wurde das Berliner Landesamt für Verfassungsschutz aufgefordert, Auskunft respektive Akteneinsıcht zu gewähren.

\section{Vom Auskunftsbegebren gegenüber dem BfV bis zur mißlungenen Klage vor dem VG Köln}

Zwischen dem Redaktionsanwalt und dem Bundesamt für Verfassungsschutz begann der übliche Schriftwechsel. Mit Datum 31. Juli 1989 teilte das BfV mit, daß es bei Wahrnehmung seiner gesetzlichen Aufgaben festgestellt habe, daß Mitglieder und Angehörige linksextremistischer Organisationen sich an der Zeitschrift $» B u ̈ r-$ gerrechte \& Polizei (CILIP) beteiligten, es aber unter Verweis auf $\mathbb{1} 3$ Abs. 2 des zu jener Zeit geltenden BDSG von der Pflicht zur Auskunft befreit sei und also eine „darüber hinausgehende Auskunft nicht erteilt werden « könne. Nur soweit hat das $\mathrm{BfV}$ in fürsorglicher Manier "Auskunft « gegeben, als es versicherte, daß die »bloße Mitarbeit « bei "Bürgerrechte \& Polizei (CILIP) « nicht als Betätigung im Sinne von $\$ 3$ BVerfSchG gesehen werde, so daß insoweit keine Speicherung beim BfV vorhanden sei. Nur, was ist »bloße Mitarbeit « an einer extremistisch unterwanderten Zeitschrift? Eine Antwort hierauf wurde trotz flehentlicher Nachfrage nicht gegeben.

Der Widerspruch unseres Anwalts wurde vom BfV mit Schreiben vom 17. November 1989 zurückgewiesen und in der Rechtsmittelbelehrung auf den Klageweg verwiesen. Daraufhin wurde am 20. Dezember 1989 beim VG Köln Klage auf Auskunft wegen ermessensfehlerhafter Entscheidung des BfV erhoben.

Parallel hierzu lief das Auskunftsbegehren gegenüber dem Berliner LfV. Eine neue Regierungskoalition, die sich mit einem gewaltigen Berg an VfS-Skandalen auseinandersetzen mußte, hatte inzwischen für Berlin eine neue Situation in dieser Frage geschaffen. Prinzipiell - so eine Entscheidung des damaligen Innensenators Pätzold - sollte das Landesamt für VfS Auskunft geben. Und so konnten Redaktionsmitglieder u.a. am 27. März 1990 in Kellerräumen unter Aufsicht in Kopien (von zuvor angefertigten und dann von Mitarbeitern des LfV bearbeiteten Kopien) ihrer Dossiers begrenzte Einsicht nehmen. ${ }^{?}$

Würde man als Kriterium für die Schnüffeltätigkeit die Richtigkeit und Vollständigkeit der gesammelten Daten annehmen, dann bräuchte man sich in dieser Republik keine zwei Sekunden mehr Sorge machen um das demokratie-bedrohliche Potential

\footnotetext{
6 Vgl. Seifert, Jürgen, Hohettliche Verrufserklärungen? Verfassungsschutzberıchte von Bund und Ländern Im Vergleıch, in: Vorgänge 1982,21 Jg., H. I (55), S. 46-60.

7 Zum Verfahren in Berlin vgl. Diederichs, Otto, "Auf Ihr Auskunftsersuchen teilen wir Ihnen mit ...., in: Bürgerrechte \& Polizeı (CILIP) 1990 (H. 36 C), S. 75 ff.
} 
der Ämter für »Verfassungsschutz « angesichts der kruden Mischung, die sich dort

den Einsichtnehmenden darbot. Wenn es nicht so ernst wäre: Ein Mitherausgeber und Autor konnte nun erstaunt seine Daten einsehen, obwohl er genau 13 Monate zuvor auf eine Auskunftsanfrage die schriftliche Auskunft erhalten hatte, daß er zu keinem Zeitpunkt vom Amte beobachtet worden sei und über ihn keine Erkenntnisse in Akten und keine Daten in Dateien vorhanden gewesen wären oder noch seien. Und ein anderer Kollege erfuhr aus den Akten, daß eın Spitzel ihn 1978 zum Mitglied einer maoistischen Partei gemacht hatte, während er in anderen - ihm nicht vorgelegten Akten - in dieser Zeit zum AL-Mitglied geworden war. Beides traf nicht zu. Aber dies ist ja gerade die unheilvolle Wirkung dieser Ämter: Auf Grund der mangelnden Überprüfbarkeit ihrer Informationen kommt es auf Richtigkeit, Vollständigkeit, Relevanz etc. gar nicht an. Was zählt, ist die Definitionsmacht der Ämter. Allein die Tatsache der Speicherung führt zur »verfassungs«schützerischen Kontaminierung wie im hier berichteten Fall der Verrufserklärung einer Zeitschrift.

Doch zurück zum Gerichtsverfahren:

In seiner Stellungnahme zum Klagebegehren teilte das BfV mit Datum 21. Juni 1990 mit der schon bekannten Argumentation mit, daß es beim mündlichen Tcrmin beantragen werde, die Klage abzuweisen. Auch dic Entgegnung unseres Anwalts auf die Klageerwiderung des Bundesamtes verhalf dort zu keinen neuen Einsichten, so daß es am 22. Februar 1991 vor dem VG in Köln zum Termin kam. Inzwischen war das oben genannte neue BVerfSchG in Kraft getreten.

Das Gericht lcgte den Klägern auf Grund der neuen Rechtslage nahe, den Rechtsstreit in der Hauptsache für erledigt zu erklären, wenn die Vertreterin des BfV sich bereıt erkläre, auf Grundlage der nun ab 1. Januar r99 r geltenden neuen Auskunftsregelung über das Auskunftsbegehren neu zu entscheiden. Im Beschluß der 20. Kammer des VG Köln vom 22.2. I99 I 1st zu lesen:

"Das Gercht weist darauf hin, daß Rechtsgrundlage für das Begehren nunmehr $\mathbb{S}$ is Bundesverfassungsschutzgesetz n. F. (BVerfSchG) ust. Nach Auffassung der Kammer spricht sehr viel dafür, daß in diesem konkreten Fall die Voraussetzungen nach $\mathbb{S}$ is Abs. I BVerfSchG, nämlich der Hinwers auf emen konkreten Sachverhalt und ein besonderes Interesse an emer Auskunft aufgrund der Vorgeschichte von den Klägern hinretchend dargelegt worden seven. Eine Entscheidung $t m$ Sinne des Abs. 2 dieser Vorschrift ust bisher von der Beklagten nucht ergangen.

Deshalb regt das Gericht an, daß die Beklagte sich verpflichtet, kurzfristzg über den gestellten Antrag der Kläger gemäß Is BVerfSchG zu entscherden.

Die Vertreterin der Beklagten erklärt:

Ich hebe meine Bescherde, die aufgrund der alten Rechtslage ergangen sind, vom 31. Juli 1989, 17. und 20. November 1989 anf, soweit darn die Auskunft verwetgert worden ist.r" $^{8}$

Frohgemut eilte der Kläger nach Berlin, mißmutig zahlte er die bisher angelaufenen Verfahrens- und eigenen Anwaltskosten, die das Gericht jeder Partei selbst zu tragen auferlegt hatte.

\section{Neue Rechtslage - alte Praxis}

Auf den Tag 3 Monate später - wohl im Bestreben, die anwaltlich bereits angekündigte Untätigkeitsklage zu vermeiden -, am 22. Mai 199I, brachte das BfV seine neuc 
Entscheidung auf den Weg. Mitgeteilt wurde, daß die von den Klägern vorgetragene Beteiligung an der Zeitschrift als ein "Hinwets auf einen konkreten Sachverhalt im Sinne des $\int_{15} A b s$. I $B$ VerfSch $G$ « in Betracht komme, die Kläger aber einen darüber hinausgehenden anderen Sachverhalt, »der em Tätızwerden des BfV im Rabmen semes gesetzlichen Auftrages nach $\mathbb{I} 3$ Abs. I BVerfSch $G$ bätte auslösen können ", nicht vorgetragen habe. »Unter Berücksuchtıgung dieser Sach-und Rechtslage kann das BfV eine andere als die bisher schon erteilte Auskunft nucht geben."

Die Antwort zeugt von Konsequenz: Wenn schon die bloße "Mitarbeit« an der Zeitschrift »Bürgerrechte \& Polizei (CILIP) « keinen Erfassungsgrund gibt, die auskunftsuchenden Herausgeber und Autoren darüber hinaus nichts vorgetragen haben, dann fehlt es an einem darüber hinausgehenden konkreten Sachverhalt, der ein Auskunftsverlangen legitimierte. In anderen Worten: Wer Besorgnis über eine Speicherung im Zusammenhang mit der Zeitschrift und amtlich verkündeter extremistischer Unterwanderung habe, müsse sein Auskunftsbegehren schon mit entsprechenden eigenen Extremismus-Nachweisen unterfüttern. Dies heißt in aller Konsequenz: Wenn der um Auskunft Ersuchende nicht seine politische Vita dem Amte en detail vorlegt, so daß das Amt anhand dieser Mitteilungen erkennen kann, ob es hätte tätig werden müssen - wenn es nicht bereits tätig geworden war --, dann gibt es keine Auskunft. Der Auskunftsbegehrende soll mithin das Amt mit eventuell sensiblen persönlichen Daten beliefern, will er eine kleine Chance auf Auskunft erhalten. Damit verkehrt sich der Auskunftsanspruch gegenüber dem Amte zur Verpflichtung zur Selbstdenunziation - so die Rechtsauffassung des BfV.

Abschließend tröstet das BfV mit dem Hinweis:

¿Aus unseren Scbriftsätzen in den vergangenen Verfabren ging bereits bervor, daß der zur Begründung ibrer Auskunftsersuchen dargelegte Sachverhalt nicht die Annahme eines datenschutzrechtlich relevanten Vorgangs in unserem Hause rechtfertigte und die Befürchtung konkreter Nachteile durch eine eventuell unrichtige oder unzulässıge Datenspeıcherung nicht hinreıchend da.getan sel."

Wie soll man enne »unrichtige oder unzulässige Datenspeicherung e erkennen, solange man keinen Einblick darin hat, was da gespeichert worden ist? Und wie lassen sich die konkreten Nachteile für enne Zeitschrift beweisen, von der die Bundesregierung mit aller Amtsautorität erklärt, daß sie »extremıstisch unterwandert« sei?

5. „So muß gewährlezstet sem, daß die Auskunftsverwergerung der Regelfall tst « - deutliche Worte eines Präsidenten

Am 13.Juni 1991 erfolgte im niedersächsischen Landtag eine Anhörung zum Entwurf eines „Gesetzes zur Neuregelung des Verfassungsschutzes Im Lande Niedersachsen «. Der Präsident des BfV legte seine Position mit Datum vom 3 r. Mai I99 I schriftlich dar.9 Zur Auskunftsregelung des niedersächsischen Entwurfs erklärte er:

"Die Regelung der Auskunftserteilung an Betroffene in $\mathbb{I} 12$ des Gesetzesentwurfs der Landesregierung trägt den berechtıgten Interessen des Verfassungsschutzes nicht in dem erforderlichen Maße Rechnung. So muß gewäbrlerstet sein, daß die Aus-

\footnotetext{
9 Bundesamt für Verfassungsschutz, An das Niedersächsısche Innenmınısterıum, betr: Entwurf eınes Gesetzes zur Neuregelung des Verfassungsschutzes Im Lande Niedersachsen; hıer: Stellungnahme des Präsıdenten, Köln den 31. Ma1 1991, 24 S.
} 
kunftsverwetgerung der Regelfall ist, selbst wenn keine Erkenntnisse zum Anfragen-

den vorliegen. *

Es folgt ein zweiseitiger Argumentationskatalog, mit dem diese Position bekräftigt wird. Hier heißt es u. a.:

"Informationen über Extremisten stammen teils aus öffentlich zugänglichen, teils aus gehermbaltungsbedürttigen Quellen. Ber der Auskunftserteilung können nur die offenen, die Spezcherung allein nicht tragenden Randerkenntnisse mitgeteilt werden. Diese würden bei einer gerichtlichen Überprüfung des Auskunftsbescherdes $u$.U. nicht ausreachen und der Verfassungsschutz zur Löschung angehalten werden. " Darum geht's - sich gerichtlicher und sonstiger Kontrolle um jedem Preis zu entziehen.

Für den hier vorgetragenen Fall ist folgende, in diesem Katalog niedergelegte Position von Interesse:

"Ein besonderes Interesse des Petenten an einer Auskunftserteilung wird regelmäßig in folgenden Fällen vorliegen:

... ber Kenntnis der Spezcherung, z. B. durch Nennung in veröffentlichten Berichten oder aufgrund von Indiskretionen. "

Nur, selbst daran hält sich das Bundesamt im vorgetragenen Falle nicht. Wie der in Berlin offiziell ermöglichte Einblick in Teilunterlagen des LfV kundtat, sind mehrere der um Auskunft ersuchenden Redaktionsmitglieder im nachrichtendienstlichen Informationssystem des Bundes und der Länder NADIS erfaßt. Aber selbst diese, durch keine Indiskretion, sondern höchst offiziell in Erfahrung gebrachte und dem Bundesamt im Auskunftsersuchen mitgeteilte Information sowie die "Indiskretion« der Bundesregierung in ihrer parlamentarischen Antwort vom 4. April 1989 reichten dem Amt im vorliegenden Falle nicht aus. Schließlich argumentiert der Präsident des Bundesamtes in dieser Stellungnahme, daß eine Auskunftsregelung das Bundesamt und die übrigen Landesämter vom Informationsaustausch mit anderen Nachrichtendiensten abzuschneiden drohe. Und gewiß: Auch im geheimdienstlichen Internationalismus gilt, daß eine Hand die andere wäscht, daß der eine nur liefert, wenn der andere mit eigenen Informationen zahlt. Auf dem Weltmarkt der Geheimdienste gilt also »Daten frei«, sofern sie im Schacher mit Informationen nachgefragt werden - das informationelle Selbstbestimmungsrecht des Bürgers hingegen scheint nur in verfassungsrechtlichen Essays papierene Gestalt anzunehmen. Und ohne Zweifel: Das Volkszählungsurteil des Bundesverfassungsgerichts ist ein herrliches, demokratietheoretisch prachtvoll argumentierender Essay. Was bleibt? Eine erneute Klage, u. U. eine neue Verhandlung vor dem VG Köln mit Termin in ca. 2 Jahren. Sollte die Klage erfolgreich sein, wird das Bundesamt den Instanzenzug bis zum Bundesverwaltungsgericht nutzen. Der letzte Termin kann für das Jahr 2000 kalkuliert werden. Bis dahin werden zwischen der hoheitlichen Verrufserklärung und dem eventuellen Offenlegen der empirischen Beweisstücke für diese Verrufserklärung I3 oder 14 Jahre vergangen seın.

Oder aber: Uns ereilt das Schicksal eines bayerischen Auskunftssuchenden, der nach $4^{1 / 2}$ jährigem zähen Kampfe unseres Redaktionsanwalts im Februar d. J. vom bayerischen Landesamt für »Verfassungsschutz« endlich Auskunft - mit folgenden einführenden Worten - erhielt:

"Ungeachtet dessen ist der Beklagte im Hinblick darauf, daß der Kläger sich offenbar in überstetgerter Weise verfolgt füblt - was aus falschen oder verzerrten Berichten über Arbeit und Beobachtungsobjekte des Verfassungsschutzes resultieren dürfte - jetzt bereit, dem Kläger die begebrte Auskunft zu erteilen. ${ }^{10}$

10 Schreiben der Landesanwaltschaft Bayreuth vom 1.2. 1991, AZ. 1/88/638. 
Inzwischen hat sich einer der »Väter « der Auskunftsregelung im neuen BVerfSchG, MdB Burkhard Hirsch, gegenüber den Autoren aus Anlaß des ihm vorgelegten „CILIP-Falles« zum »Willen des Gesetzgebers« geäußert. »Unser Ausgangspunkt war natürlich, daß in der Regel Auskunft gegeben wird. " "

\section{Ivo Heiliger \\ Ein zweites Fehlurteil gegen Ossietzky}

Über den Wert der Aktion mag man geteilter Meinung seın.

Carl von Ossietzky ist längst als "der Dulder" (Heinrich Mann) in die Historie eingegangen. Seine Verurteilung wird ein Tiefpunkt der ohnehin wenig rühmlichen Geschichte des Reichsgerichts bleiben.

Dennoch entschloß sich seine Tochter Rosalinda, auch die juristische Rehabilitation ihres Vaters zu betreiben, und hat mit einem Antrag auf Wiederaufnahme des Verfahrens die Justiz gezwungen, Farbe zu bekennen. Der Zeitpunkt war - ohne daß jemand es bei der Antragstellung geahnt hätte - gut gewählt. Das Prestige des Reichsgerichts hat durch die mittlerweile weit verbreitete Kenntnis seiner Urteile aus der Nazi-Zeit erheblich gelitten, und kaum eine Person der jüngeren Geschichte ist in Deutschland Ost und Deutschland West so gleichermaßen unumstritten wie der Friedensnobelpreisträger Carl von Ossietzky.

Zur Erinnerung:

Der Publizist Carl von Ossietzky, Herausgeber der "Weltbühne«, war am 23. November 1931 vom Vierten Strafsenat des Reichsgerichts wegen Verrats militärischer Geheimnisse nach $\mathbb{1}$ Abs. 2 des sog. Spionagegesetzes vom 3. Juni 1914 zu 18 Monaten Gefängnis verurteilt worden. Die »Weltbühne« hatte im März 1929 unter dem Titel „Windiges aus der deutschen Luftfahrt einen Beitrag des Luftfahrtexperten Walter Kreiser über die (nach dem Versailler Vertrag verbotenen) Luftfahrtaktivitäten der Reichswehr veröffentlicht, und diesen nahm die Reichsanwaltschaft zum Anlaß, dem ungeliebten Mahner gegen Militarismus und heraufziehenden Faschismus den Prozeß zu machen. Zuständig für Hoch- und Landesverratsverfahren war damals der Reichsanwalt Paul Jorns, gerichtsnotorischer Begünstiger der Mörder Karl Liebknechts und Rosa Luxemburgs und nachmaliger Chefankläger beim neuerrichteten Volksgerichtshof. Dem Gericht saß der Reichsgerichtsrat Baumgarten vor, der im Jahr zuvor in einem Hochverratsprozeß gegen dre junge Reichswehroffiziere Hitler die Gelegenheit gegeben hatte, seinen »Reinigungseıd « zu leisten, d. h. er hatte Hitler als sachverständigen Zeugen zu der Frage geladen, ob die NSDAP eine umstürzlerische Partei sei. Als Sachverständiger der Reichswehr trat im »Weltbühnen «-Prozeß der skandalumwitterte Major Himer auf, Organisator der sog. Schwarzen Reichswehr, Drahtzieher der illegalen Rüstung. Die Vorstellung der gespenstischen Akteure dieses Prozesses scheint mir für das Verständnis seines Verlaufs und vor allem seines Ergebnisses von Bedeutung zu sein. Die Verteidigerbank hätte dagegen nicht besser besetzt sein können. Mit Max Alsberg, Alfred Apfel, Rudolf Olden und Kurt Rosenfeld stand Ossietzky ein Verteidigerquartett zur Seite, wie es seither wohl kein besseres gegeben hat. Es hat ihm wenig genützt.

I I So Hirsch in einem Schreiben vom 1. 11. 1991. 